\title{
Screening in treatment programs for Fetal Alcohol Spectrum Disorders that could affect therapeutic progress
}

\author{
Therese M. Grant ${ }^{1}$, Natalie Novick Brown', J. Christopher Graham², Nancy Whitney², Dan Dubovsky ${ }^{3}$, \\ and Lonnie A. Nelson ${ }^{4}$ \\ ${ }^{1}$ University of Washington School of Medicine, Department of Psychiatry and Behavioral Sciences, Seattle, WA, United States \\ ${ }^{2}$ University of Washington Health Sciences Administration, Alcohol and Drug Abuse Institute, Seattle, WA, United States \\ ${ }^{3}$ Substance Abuse and Mental Health Services Administration (SAMHSA) Fetal Alcohol Spectrum Disorders Center for Excellence, \\ Rockville, MD, United States \\ ${ }^{4}$ University of Washington School of Public Health, Department of Health Services, Seattle, WA, United States
}

\begin{abstract}
Aims: While structured intake interviews are the standard of care in substance abuse treatment programs, these interviews often do not screen for cognitive impairments, such as those found in fetal alcohol spectrum disorders (FASD) and other brain-based developmental disorders. The research reported here supports a brief interview protocol, the Life History Screen (LHS), that screens clients unobtrusively for adverse life-course outcomes typically found in FASD, so as to guide follow-up assessments and treatment planning.
\end{abstract}

Design: Two-group observational study.

Setting: A three-year case management intervention program in Washington State for high-risk women who abuse alcohol and/or drugs during pregnancy.

Participants: Group 1: No prenatal alcohol exposure $(N=463)$; Group 2: Diagnosed with FASD (Fetal Alcohol Syndrome, Alcohol Related Neurodevelopmental Disorder, fetal alcohol effects, or static encephalopathy) by a qualified physician $(N=25)$, or suspected of having FASD (reported prenatal alcohol exposure and displayed behaviors consistent with a clinical diagnosis of FASD) $(N=61)$.

Measures: The Addiction Severity Index (ASI) was administered to participants at intake. We analyzed eleven ASI items that corresponded to questions on the LHS in order to assess the potential of the LHS for identifying adults with possible FASD. The Life History Screen itself was not administered.

Findings: Analysis of group differences between the diagnosed FASD and suspected FASD groups supported our decision to collapse the two groups for the main analysis. The Life History Screen shows promise as an efficient pre-treatment screen, in that core items are significantly associated with FASD group membership on factors involving childhood history, maternal drinking, education, substance use, employment, and psychiatric symptomatology.

Conclusions: The Life History Screen may have utility as a self-report measure that can be used at the outset of treatment to identify clients with cognitive impairments and learning disabilities due to prenatal alcohol exposure.

While approximately 2.6 million people enter substance abuse treatment in the United States every year (National Institute on Drug Abuse [NIDA], 2011), only about half of these individuals complete treatment (Substance Abuse and Mental Health Services Administration (SAMHSA), 2012). Treatment providers often attribute this treatment failure to clients' poor motivation; clients tend to blame their providers. Both attributions may be inaccurate. An alternative reason for treatment disruption may be that clients have neurocognitive deficits and learning disabilities associated with unidentified medical conditions such as fetal alcohol spectrum disorders (FASD). In fact, FASD is likely an issue for a large percentage of substance abuse treatment clients, as it is well-established that prenatal alcohol exposure predisposes adolescents and adults to alcohol and drug problems (Baer, Barr, Bookstein, Sampson, \& Streissguth, 1998; Baer, Sampson, Barr, Connor, \& Streissguth, 2003; Streissguth, Barr, Kogan, \& Bookstein, 1996; Yates, Cadoret, Troughton, Stewart, \& Giunta, 1998).

Correspondence: Therese Grant, Ph.D., University of Washington, 180 Nickerson St. Suite 309, Seattle, WA 98109, United States. Telephone: +1 206 543 7155; Fax: +1 206685 2903; E-mail: granttm@u.washington.edu

Financial support: Supported by the Washington State Department of Social and Health Services Division of Behavioral Health and Recovery, contracts $0965-69005$ and 1165-29940

Keywords: fetal alcohol syndrome, fetal alcohol spectrum disorders, substance abuse treatment, screening 
FASD conditions are caused by prenatal alcohol exposure (PAE) in amounts sufficient to cause brain damage. FASD has been referred to as a "hidden disability" because many adults with FASD are unaware of their condition (Bertrand et al., 2004), although they may have had problematic life experiences stemming from brain damage caused by PAE. Learning difficulties and other neurodevelopmental problems common in FASD are due to underlying cognitive deficits (e.g., intellectual, attention, memory, sensory integration, executive functioning) that conjointly reduce ability to attend to, process, store, retrieve, and apply information (Kodituwakku, 2007; Kodituwakku, Kalberg, \& May, 2001). Based upon epidemiological estimates that up to five percent of the population in the United States may suffer from an FASD (May et al., 2009) and research indicating that at least a third of these individuals have substance abuse problems (Streissguth et al., 1996), it is likely that a sizable number of adults with undiagnosed FASD enter substance abuse treatment programs each year, having brain-based disabilities that significantly impair their ability to learn and benefit from that treatment. Consistent with poor treatment outcomes predicted by such a scenario, an initial study at the University of Washington that compared substance treatment completion rates of female clients found that women with FASD and those with PAE/suspected FASD had higher treatment failure rates than did women without PAE (Grant, Brown, Graham, \& Ernst, in press). We suggest that what the women in these FASD/PAE groups may have had in common were cognitive impairments and associated learning problems due to their prenatal exposure histories. If a substantial number of participants in treatment programs do in fact suffer from FASD, information obtained during treatment intake would be useful in individualizing treatment planning so as to maximize the potential for success. If such screening indicates problems, we suggest that referral for neuropsychological assessment (to identify impediments that could compromise treatment progress) and modifications in treatment planning could improve outcomes. Grant and colleagues (in press) and Sparrow, Grant, Connor and Whitney (2013) describe clinical case examples and provide recommendations for accommodations to address some of the impairments associated with FASD that limit substance abuse treatment success.

Identifying conditions involving brain-based disabilities like FASD is challenging if there are no obvious physical characteristics to signal the underlying medical condition (e.g., Bertrand et al., 2004). However, FASD conditions that do not involve the distinct facial abnormalities characteristic of Fetal Alcohol Syndrome (FAS), such as partial FAS and Alcohol Related Neurodevelopmental Disorder (ARND), involve similar neurodevelopmental profiles (Hoyme et al., 2005). Methodologies designed to screen for FASD typically involve informant-based protocols that ask caregivers, family members and friends (or probation officers, in the case of youth in the juvenile justice system) to rate or score individuals on life history, behavioral difficulties and other factors associated with FASD. Several such screens have been developed for children and youth, according to a review by the Public Health Agency of Canada (2011). Goh and colleagues (2008) critically reviewed seven of these measures for children and youth, three of which (Asante Centre youth justice, facial dysmorphology, and Medicine Wheel screens) were considered possibly suitable for adult screening, with appropriate modifications. In their present formats, the behavioral screens in this group suffered generally from incomplete psychometric study, particularly with respect to accurately differentiating between children with FASD and children with other conditions (e.g., Attention Deficit Disorder and conduct disorder).

According to the Public Health Agency of Canada (2011), an informant-based measure designed to detect frontal lobe impairments that was not included in Goh's analysis (Grafman \& Litvan, 1999) appeared suitable for adults as well as children, but was restricted in scope to executive function deficits, and was not specific to FASD. Another screen, the Fetal Alcohol Behavior Scale (FABS), not only was considered suitable for adults as well as children but also appeared to have undergone more rigorous developmental analysis than any of the screens noted above. Administered to caregivers, the FABS (Streissguth, Bookstein, Barr, Press, \& Sampson, 1998) demonstrated good psychometrics in research settings. To date, the FABS has not been tested in clinical contexts, where performance data would substantiate its usefulness (Boland, Burrill, Duwyn, \& Karp, 1998).

High sensitivity (ability to screen positively those individuals who have the condition) and high specificity (ability to screen negatively those who do not have the condition) are indicative of an accurate screening tool, regardless of information source. Presumably, the more items endorsed on such screens, the greater the likelihood of FASD. In theory, using self-reported screening data from individuals who may have FASD is problematic because the accuracy of such information could be substantially reduced by memory difficulties, suggestibility, executive function problems, and other cognitive deficits characteristic of the disorder. Indeed, substance abuse questionnaires and psychological tests suffer from similar reliability problems. In our clinical experience we have found an "endorsement bias" in individuals with FASD; this may stem from a social deficit described as hyper-suggestibility (Brown, Gudjonsson, \& Connor, 2011), which comes into play when providers or assessment questionnaires suggest possible symptoms or problems to individuals inclined to acquiesce to authority figures. Nonetheless, while information from unimpaired collateral informants may be more accurate than that selfreported by individuals with FASD, most treatment programs, for a variety of reasons, do not involve such informants in the intake process. Consequently, while it is understood that self-reported data from individuals with possible brain damage may produce inaccurate histories, such information often is the only data available at time of treatment. In the Methods section below, we provide information on the steps we took to overcome these limitations during interviews for the present study. 
The purpose of this article is to introduce the Life History Screen (LHS), a self-report tool designed to quickly screen for brain-based difficulties that might interfere with treatment. Although the LHS itself was not administered (as we describe below), the current study represents a preliminary step in determining the utility of the LHS for screening adults entering treatment programs. We emphasize that the purpose of the LHS is not to elicit a detailed history, but rather to screen efficiently for the possibility of cognitive problems that could interfere with treatment success. It should also be emphasized that screening is not diagnosis. Thus, individuals who test positive on an FASD screen should be referred for evaluation. Nevertheless, since diagnostic capacity for FASD in adults is severely limited in most treatment settings, the results of this screen can inform modifications in treatment approaches for those who screen positive.

\section{Methods}

\section{Participants}

Data presented in this study are from 549 women enrolled in the Washington State Parent-Child Assistance Program (PCAP) from November 1997 through July 2011. The PCAP model has been described in detail elsewhere (Ernst, Grant, Streissguth, \& Sampson, 1999; Grant, Ernst, Streissguth, \& Stark, 2005). In brief, the program is a three-year home visitation and case management intervention for high-risk women aged 18 and higher who abuse alcohol and/or drugs during pregnancy. Standard PCAP eligibility criteria are: 1) currently pregnant, or up to six months postpartum; 2) self-reported heavy alcohol and/or illicit drug use during the index pregnancy; and 3) ineffective or nonexistent engagement with community services.

For the present study, we categorized cases into one of two groups:

Group 1: No PAE/FASD $(N=463)$. Women in Group 1 were enrolled under standard PCAP eligibility criteria. None self-reported having a mother who drank alcohol during pregnancy (during the intake interview, all responded "no" to the query "Did your mother drink alcohol when she was pregnant with you?”). Client PAE information was also not reported by the client or by family members during the course of the intervention.

Group 2: Diagnosed with an FASD or suspected FASD (N = 86). In 1999, PCAP expanded its eligibility to allow enrollment of women who had been diagnosed with an FASD (including FAS, ARND, fetal alcohol effects, or static encephalopathy) at some time prior to being referred to PCAP. Twenty-five women were enrolled under this criterion. All had been diagnosed by a qualified physician or FASD multidisciplinary diagnostic team, which was verified by clinic records. Their average age at time of FASD diagnosis was 16 years (range: birth to 45 years); 21 had children or were pregnant at intake, and four had no children. Another 61 women had suspected FASD. A majority of these latter clients reported PAE during the intake interview (65.9\% answered "yes" to the query "Did your mother drink alcohol when she was pregnant with you?"). For the rest, following intake and during the course of intervention, PAE information was either self-reported and/or reported by a family member. All women in Group 2 reported histories of social problems and displayed adult behaviors consistent with a clinical diagnosis of FASD. Behavioral observations were documented by PCAP clinical supervisors on a structured case form designed for that purpose. All forms were reviewed by author TMG.

Institutional Review Board approval was obtained from the Washington State Institutional Review Board, and a certificate of confidentiality was obtained from the federal Department of Health and Human Services.

\section{Measures}

Addiction Severity Index (ASI). At intake into the threeyear PCAP intervention, all participants were interviewed using the ASI (McLellan et al., 1992), a self-report instrument widely used for clinical assessment and research purposes. In 1997, PCAP researchers developed questions to supplement the ASI regarding childhood history, community services, family planning, and substance use during pregnancy.

ASI interview procedures. PCAP does not involve informants in the intake process, for several reasons. For example, most women enrolled in the program had been alienated from family members for years, and had little or no social support (Grant, Huggins et al., 2011; Grant, Jack, Fitzpatrick, \& Ernst, 2011). Most were not raised by their biological parents, and it would have been difficult, if not impossible, to locate their birth mothers. Regarding PAE, while child protective services, adoption records, and/or court records may contain relevant information, documented evidence of PAE in contemporaneous records is rare (Brown, Wartnik, Connor, \& Adler, 2011). Thus, self-reported PAE was the only available information during intake, as is likely the case in most adult treatment programs.

In light of the acknowledged weaknesses of client-based information, efforts were made to enhance self-report accuracy. Intake interviewers were licensed mental health or social work PCAP clinicians who had extensive experience working with women who had trauma histories. Interviewers were not blind to client FASD status, because questions about medical diagnoses were asked on the standardized ASI. The intake interview was the first extensive interpersonal interaction with a member of the PCAP team, and served as a therapeutic process where clients could describe their personal histories and identify areas where they needed help. Interviewers explained that they would be asking sensitive questions about personal and biological family history so that the PCAP team could better understand the challenges the clients had experienced and how best to help them. Interviewers did not rush clients, were respectful, and urged clients to stop and ask questions if they had any uncertainty or concern. When clients endorsed an item, interviewers prompted for details. 
When clients exhibited resistance or defensiveness, interviewers provided reassurance that no assumptions or judgments were being made and that data would not be used to "blame" anyone, but rather to enhance treatment planning.

\section{Construction of the Life History Screen (LHS) (Table 1)}

In guidelines prepared for the National Center on Birth Defects and Developmental Disabilities in the United States, the Centers for Disease Control and Prevention (Bertrand et al., 2004) suggested screening criteria for referring children for full FASD assessment. Other than the facial and growth abnormalities included in these guidelines - which often become normalized by adulthood, if they are present at all (Bertrand et al., 2004) — there are two areas that appear to provide appropriate criteria for adult screening in cases involving either known or unknown PAE: social and family history and Central Nervous System (CNS) abnormalities. Regarding the latter, Boland and colleagues (1998) describe a general FASD behavioral phenotype for adolescents and adults: a mix of primary cognitive and neurodevelopmental deficits (e.g., memory, attention, executive function, adaptive behavior) and associated disabilities and related issues (e.g., easily victimized, unfocused and distractible, difficulty handling money, trouble understanding consequences and learning from experience, problems perceiving social cues, poor frustration tolerance, inappropriate sexual behavior, mental health problems, trouble with the law) that could potentially be mitigated by treatment. Together, the screening guidelines suggested for the CDC (Bertrand et al., 2004) and behavioral phenotype described by Boland and colleagues (1998) provide the rationale for the 27 items included in the LHS. For each category of items selected for the LHS, at least four independent studies found an association between the category and FASD (see Table 1).

The 27-item LHS itself was not administered to participants in this study. Instead, the Addiction Severity Index (ASI) interview was conducted with both groups of study participants, as described above. The 11 ASI items that correspond to items on the LHS were subjected to preliminary statistical analysis to assess their potential utility in identifying adults with possible FASD. Thus, data are not available for LHS items that do not have corresponding variables on the ASI (e.g., specific problems in school).

Use of the LHS does not currently involve an overall scoring system or cut-point because the present study is only the first step in establishing the scale's psychometric properties (see "Establishing LHS Psychometrics" below). Cognitive impairments in FASD tend to be diffuse and dependent upon many factors (Abel, 1998; Bertrand et al., 2004; Bookstein \& Kowell, 2010; Lipinski et al., 2012; Stratton, Howe, \& Battaglia, 1996). Therefore, attached to each screening question is the specific response that indicates a risk or "red flag" for possible cognitive impairment. The greater the number of flags, the more attuned the clinician should be to the idea that FASD and associated cognitive impairment may exist.

\section{LHS Screening Protocol}

The LHS is designed as a structured screening instrument that can be embedded within a treatment program's existing intake interview protocol and administered by service providers and clinicians to clients who have completed detoxification and are entering treatment. The screen, which requires approximately 15 minutes to administer, should be introduced as early as possible in the treatment process, so that strategies may be implemented that will help avoid treatment failure. The protocol for administration is described in the Appendix. Based upon self-report, LHS questions are framed within the context of a client's customary behavior. Thus, in the case of clients presenting for substance abuse treatment, "customary" would not include behaviors that only manifest while under the influence of alcohol or drugs. Although clinicians may have reservations about asking clients to divulge personal information and family history, fearing that if such probing is too intrusive it might negatively impact the therapeutic relationship, we have found that when the need for such questioning is explained non-judgmentally and with compassion, clients usually respond positively and candidly.

\section{Data Analyses}

We justified collapsing the diagnosed FASD $(N=25)$ and suspected FASD ( $N=61$ ) groups for the main study analysis by comparing between-group data on intake demographics and on the LHS items available on the ASI. For continuous variables we used independent samples $t$ tests, and for other variables (all categorical) we used Fisher's exact test for dichotomous variables and Pearson's Chi-Square test for the multi-category race variable. All were two-tailed significance tests.

For the main study, we examined the potential utility of the LHS in identifying clients with FASD by comparing Group 1 (no PAE/FASD) vs. Group 2 (diagnosed/suspected FASD) on their data from the 11 ASI items corresponding to items on the LHS. We used Fisher's exact test for significance testing of categorical comparisons, and for continuous variables we used independent samples $t$-tests (for unequal sample sizes and unequal variances, Levene's test was used if warranted). All significance tests were one-tailed tests, given the directional hypotheses associated with the screening instrument. We made no correction for testing of multiple hypotheses because the intent of the analysis was descriptive. Thus, resulting $p$-values are provided solely for informational purposes.

We also conducted a Receiver Operating Characteristic (ROC) curve analysis to determine the optimal cut-point for the total of the eleven LHS items corresponding to the ASI (i.e., the cut-point yielding the most favorable balance between sensitivity and specificity regarding detection of individuals with probable FASD), and to provide an initial estimate of classification accuracy. 
Table 1

Life History Screen (LHS). Note: “Red flag response” indicates risk of possible cognitive impairment

\begin{tabular}{l} 
Life History Item \\
\hline Childhood History \\
Were you raised by someone other than your \\
biological parents? ${ }^{\text {a }}$ \\
How many living situations did you have while \\
you were growing up (up to the age of 18)? \\
Prompt: living with your parents, relatives, \\
foster homes, juvenile justice setting, etc. \\
Maternal Alcohol Use \\
Did your mother ever have a problem with \\
drinking alcohol? \\
Prompt: "Can you tell me about this?" \\
Did she drink alcohol when you were young? a \\
Did she drink alcohol while she was pregnant \\
with you? \\
Prompt: If the client doesn't know, ask "Has \\
anyone ever said anything to you about your \\
mother's drinking? Is there anyone around who \\
knew your mother when she was pregnant with \\
you?"
\end{tabular}

Education

What's the highest grade in school you completed?

If you didn’t finish school, why did you leave?

Were you ever in "special ed" or did you get any kind of special help in school?

Criminal History

Were you ever arrested? ${ }^{\mathrm{a}}$

Substance Use

In what grade (or at what age) did you start using alcohol or drugs? ${ }^{\text {a }}$

Employment and Income

What's the longest time you've worked at the same job? ${ }^{\mathrm{a}}$

Have you ever received income from SSI? ${ }^{a}$

\section{Red Flag Response}

Basis for Screening Factor (Source)

- Clark, Lutke, Minnes, \& Ouellette-Kuntz, 2004

- Löser, Bierstedt, \& Blum, 1999

- Streissguth et al., 1991

- Streissguth, Barr, Kogan, \& Bookstein, 1996

(yes)

(more than 2)

(Red flag response)

(yes)

(yes)

(yes)

(Red flag response)

(10th or lower)

(too hard, bored, got kicked out, or another reason)

(yes)

(Red flag response)

(yes)

(Red flag response)

(before age 12, or alcohol/ drug use began after school problems started)

(Red flag response)

(less than one year)

(yes)
- Centers for Disease Control and Prevention (CDC), 2004

- Jones \& Smith, 1973

- Jones, Smith, Ulleland, \& Streissguth, 1973

- Streissguth et al., 1991

- Clark, Lutke, Minnes, \& Ouellette-Kuntz, 2004

- Löser, Bierstedt, \& Blum, 1999

- Spohr, Willms, \& Steinhausen, 2007

- Streissguth, Barr, Kogan, \& Bookstein, 1996

- Mattson \& Riley, 2000

- Roebuck et al., 1999

- Streissguth et al., 1991

- Streissguth et al., 2004

- Alati et al., 2008

- Baer, Barr, Bookstein, Sampson, \& Streissguth, 1998

- Baer, Sampson, Barr, Connor, \& Streissguth, 2003

- Barr et al., 2006

- Famy, Streissguth, \& Unis, 1998

- Spohr, Willms, \& Steinhausen, 2007

- Steinhausen, 1996

- Streissguth, Barr, Kogan, \& Bookstein, 1996 


\begin{tabular}{|c|c|c|}
\hline Life History Item & Red Flag Response & Basis for Screening Factor (Source) \\
\hline Living Situations & (Red flag response) & $\begin{array}{l}\text { - Clark, Lutke, Minnes, \& Ouellette-Kuntz, } 2004 \\
\text { - Löser, Bierstedt, \& Blum, } 1999 \\
\text { - Spohr, Willms, \& Steinhausen, } 2007 \\
\text { - Streissguth, Barr, Kogan, \& Bookstein, } 1996\end{array}$ \\
\hline $\begin{array}{l}\text { As an adult have you ever lived on your own } \\
\text { (paying your own rent, etc.)? }\end{array}$ & (no) & \\
\hline Mental Health & (Red flag response) & $\begin{array}{l}\text { - Astley, Bailey, Talbot, \& Clarren, } 2000 \\
\text { - Huggins, Grant, O’Malley, \& Streissguth, } 2008 \\
\text { - O’Connor \& Paley, } 2009 \\
\text { - Roebuck, Mattson, \& Riley, } 1999 \\
\text { - Spohr, Willms, \& Steinhausen, } 2007\end{array}$ \\
\hline $\begin{array}{l}\text { Other than a substance abuse disorder, what kinds } \\
\text { of mental health disorders have you been told you } \\
\text { have? }{ }^{\text {a }}\end{array}$ & (more than one) & \\
\hline Have you ever tried to commit suicide? ${ }^{a}$ & (yes) & \\
\hline Day-to-Day Behaviors & (Red flag response) & $\begin{array}{l}\text { - Clark, Lutke, Minnes, \& Ouellette-Kuntz, } 2004 \\
\text { - Hellemans, Sliwowska, Verma, \& Weinberg, } \\
2010 \\
\text { - Kodituwakku, Kalberg, \& May, } 2001 \\
\text { - Mattson, Goodman, Caine, Delis, \& Riley, } 1999 \\
\text { - Streissguth et al., } 1998\end{array}$ \\
\hline \multicolumn{3}{|l|}{$\begin{array}{l}\text { When you were a child in grade school, did you } \\
\text { often have difficulties with any of the } \\
\text { following? }\end{array}$} \\
\hline - Concentrating and paying attention? ${ }^{\mathrm{a}}$ & (yes) & \\
\hline $\begin{array}{l}\text { - Understanding what adults were telling } \\
\text { you? }\end{array}$ & (yes) & \\
\hline $\begin{array}{l}\text { - Remembering things? } \\
\text { - As a child or as an adult have you often } \\
\text { have difficulties with any of the } \\
\text { following? }\end{array}$ & (yes) & \\
\hline - following rules and instructions & (yes) & \\
\hline $\begin{array}{l}\text { - getting along with others, arguing, or } \\
\text { fighting }\end{array}$ & (yes) & \\
\hline - being on time & (yes) & \\
\hline $\begin{array}{l}\text { - keeping enough money to last you } \\
\text { throughout the month }\end{array}$ & (yes) & \\
\hline $\begin{array}{l}\text { - doing things on the spur of the moment } \\
\text { that later you wish you hadn't done }\end{array}$ & (yes) & \\
\hline - getting really upset at little things & (yes) & \\
\hline - forgetting or missing appointments & (yes) & \\
\hline - being surprised when you get into trouble & (yes) & \\
\hline
\end{tabular}

${ }^{\text {a }}$ Data on this item was available from the ASI and is presented on Table 3.

\section{Results}

\section{Comparison of the Two Collapsed FASD Groups}

In examining differences between women diagnosed with FASD and those with suspected FASD, we found no statistically significant differences between the diagnosed FASD $(n=25)$ and suspected FASD $(n=61)$ groups with regard to mother's age (mean years 23.2 vs. 24.9 respectively), race (56.0\% White, $28.0 \%$ Native American, and $16.0 \%$ Asian, Black, or Hispanic vs. 50.8\% White, 29.5\% Native American, and $19.7 \%$ Asian, Black, or Hispanic), and marital history (never married $80.0 \%$ vs. $70.5 \%$ ) (data not presented on tables). Diagnosed women had, on average, one less child than did women with suspected FASD (mean 1.7 vs. 2.8, $p<.005$ ), and a higher proportion of women in the diagnosed group were living in permanent/stable housing compared to those in the suspected group ( $56.0 \%$ vs. $29.5 \%, p<.05)$. On the LHS items examined, we found significant differences between the diagnosed FASD and suspected FASD women in terms of reports that their mothers had an alcohol problem (95.8\% vs. $73.3 \%, p<.05$ ) and reports of having more than one psychiatric diagnosis $(8.0 \%$ vs. $39.3 \%, p<.005)$. We found no other significant differences between the two groups. However, given the small sample sizes, the statistical power of these tests was limited. 
Intake maternal demographic characteristics (Table 2) At intake, women in Group 2 (diagnosed and suspected FASD) were younger than women in Group 1 (no PAE/FASD) $($ mean $=24.4$ vs. 26.8 years, $p<.001$ ). We observed a higher proportion of Native American women in Group 2 compared to Group 1 (29.1\% vs. 13.0\%, $p<.001)$.

\section{Analysis of ASI variables corresponding to LHS items} (Table 3)

Women in Group 2 had significantly higher rates of endorsement of nine of the 11 ASI/LHS items compared to Group 1, including:

- childhood history: raised by someone other than their biological parents (64.0\% vs. $26.4 \%, p<.001)$;

- maternal alcohol use: client's mother had a problem with alcohol (79.8\% vs. 29.9\%, $p<.001)$;

- mothers drank during their childhoods $(89.5 \%$ vs. $37.7 \%, p<.001)$;

- education: 10 th grade education or lower $(48.8 \%$ vs. $33.3 \%, p<.01$ );

- substance use: client began using alcohol or drugs before age 12 (47.0\% vs. $28.1 \%, p<.001)$;
- employment: longest full-time job was less than 12 months (72.1\% vs. $48.6 \%, p<.001)$; mental health: more than one psychiatric diagnosis $(30.2 \%$ vs. $13.8 \%, p<.001)$;

- suicide attempt (45.9\% vs. $31.7 \%, p<.01)$; and

- day-to-day behaviors: history of trouble concentrating (78.8\% vs. 52.7\%, $p<.001$ ).

The two groups differed marginally on the income item "receives Social Security income" (12.8\% vs. $6.9 \%, p=$ .06 ), and there was no difference on the item "one or more arrests (with charges) since age 18" (76.5\% [354/463] vs. $76.5 \%$ [65/86], ns).

\section{ROC Analysis}

Results of the ROC curve analysis suggest that in this sample, the cut-point with the most favorable balance of sensitivity and specificity was a score of $\geq 5$ on the 11 LHS items available. A score of five or higher was associated with a sensitivity of $80.8 \%$ and a specificity of $65.5 \%$, with $67.6 \%$ of cases correctly classified. The positive likelihood ratio for this cut-point was 2.34 , and the negative likelihood ratio was 0.29 .

Table 2.

Maternal demographic characteristics at program intake

\begin{tabular}{|c|c|c|c|}
\hline Characteristic & $\begin{array}{c}\text { Group } 1 \\
\text { No PAE or FASD } \\
(N=463)\end{array}$ & $\begin{array}{c}\text { Group } 2 \\
\text { Diagnosed or } \\
\text { Suspected FASD } \\
(N=\mathbf{8 6}) \\
\end{array}$ & $p$-value ${ }^{a}$ \\
\hline \multicolumn{4}{|l|}{ Age } \\
\hline Mother's age (mean years) & 26.8 & 24.4 & $* * *$ \\
\hline \multicolumn{4}{|l|}{ Parity } \\
\hline Total number of children (mean) & 2.7 & 2.5 & ns \\
\hline \multicolumn{4}{|l|}{ Race } \\
\hline White & $296(63.9 \%)^{+}$ & $45(52.3 \%)^{-}$ & $* * *$ \\
\hline Native American & $60(13.0 \%)^{-}$ & $25(29.1 \%)^{+}$ & \\
\hline Asian / Black / Hispanic & $107(23.1 \%)$ & $16(18.6 \%)$ & \\
\hline \multicolumn{4}{|l|}{ Marital } \\
\hline Never married & $318(68.7 \%)$ & $63(73.3 \%)$ & $n s$ \\
\hline \multicolumn{4}{|l|}{ Housing } \\
\hline Permanent / Stable housing & 160 (34.6\%) & 32 (37.2\%) & ns \\
\hline
\end{tabular}

${ }^{*} p<.05 ; * * p<.01 ; * * * p<.001$

${ }^{a}$ Groups with categorical percentages significantly greater or less than what would be expected from the marginal distributions are indicated by valence superscripts ( + or - ). 
Table 3.

\begin{tabular}{|c|c|c|c|}
\hline LHS Items & $\begin{array}{c}\text { Group } 1 \\
\text { No PAE or FASD } \\
(N=463)\end{array}$ & $\begin{array}{c}\text { Group } 2 \\
\text { Diagnosed or } \\
\text { Suspected FASD } \\
(N=\mathbf{8 6})\end{array}$ & $p$-value \\
\hline Raised by someone other than biological parents & $122(26.4 \%)$ & $55(64.0 \%)$ & $* * *$ \\
\hline Client's mother had a problem with alcohol & $138(29.9 \%)$ & $67(79.8 \%)$ & $* * *$ \\
\hline Client's mother drank when client was a child & $172(37.7 \%)$ & $68(89.5 \%)$ & $* * *$ \\
\hline Educational level 10th grade or lower & $154(33.3 \%)$ & $42(48.8 \%)$ & $* *$ \\
\hline One or more arrests (and charged) since age 18 & $354(76.5 \%)$ & $65(76.5 \%)$ & ns \\
\hline Client started using substances before age 12 & $130(28.1 \%)$ & $39(47.0 \%)$ & $* * *$ \\
\hline Longest full-time job was less than 12 months & $225(48.6 \%)$ & $62(72.1 \%)$ & $* * *$ \\
\hline Receives Social Security Insurance (SSI) & $32(6.9 \%)$ & $11(12.8 \%)$ & $\mathrm{ns}^{\mathrm{a}}$ \\
\hline More than one psychiatric diagnosis & $64(13.8 \%)$ & $26(30.2 \%)$ & $* * *$ \\
\hline History of trouble concentrating & $244(52.7 \%)$ & $67(78.8 \%)$ & $* * *$ \\
\hline Suicide attempt & $147(31.7 \%)$ & 39 (45.9\%) & $* *$ \\
\hline
\end{tabular}

${ }^{*} p<.05,{ }^{* *} p<.01,{ }^{* * *} p<.001$

${ }^{\mathrm{a}} p$ - value $=.06$

\section{Discussion}

The LHS is composed of 27 items that describe life history outcomes observed in individuals who suffer from an FASD. In order to examine the potential utility of the LHS in identifying clients with FASD, this study examined 11 variables from PCAP's intake interview that paralleled LHS items, and compared responses from women in PCAP with diagnosed/suspected FASD versus those without PAE. We first conducted an analysis of group differences between the diagnosed FASD and suspected FASD groups, to support our rationale for collapsing the two groups for the main analysis. We found few differences, and these were understandable. Specifically, diagnosed women had fewer children, because they were enrolled in PCAP under the expanded eligibility criterion that didn't require them to have children. A higher proportion of women in the diagnosed group were living in permanent/stable housing compared to the suspected group, probably because adults with recognized FASD typically live dependently with family members or in other stable settings (Spohr, Willms, \& Steinhausen, 2007; Streissguth et al., 1996; Streissguth et al., 2004). A higher proportion of women in the diagnosed group reported having more than one psychiatric diagnosis, but this finding may be spurious. For example, if the FASD diagnosis was the focus of mental health treatment, additional psychiatric issues may not have been closely examined. Alternatively, the finding could be due to memory impairment; that is, it may have been the case that other psychiatric issues were identified, but the client recalled only a primary FASD diagnosis. Another explanation could be that some diagnosed women received early support for their FASD and consequently did not develop some of the mental health issues that those without support often experience, such as depression and anxiety.
Primary findings in this study indicated that most LHS questions (e.g., childhood history, maternal drinking, education, substance use, employment, and psychiatric symptomatology) were strongly associated with women having diagnosed/suspected FASD. Our finding of no difference between groups on the LHS "arrest" item was due to the high base rate of arrest in the PCAP sample (overall, 76\% reported prior incarceration, with a mean of 5.6 arrests). This study shows that even with their disability, individuals with FASD/suspected FASD are capable of endorsing carefully-worded questions that do not screen for symptoms and impairments in an obvious way. Thus, the measure may minimize the effects of conscious or unconscious self-presentation bias in FASD, thereby improving the odds of detecting cognitive impairment even when a practitioner does not have a great deal of experience with this population.

Although we did not have ASI data available to compare with every item on the LHS, the research cited on Table 1 supports our decision to include each category in the measure. For example, in a landmark study on FASD across the lifespan, Streissguth and colleagues (1996, 2004) examined characteristics and adverse life outcomes among 473 clients, including 90 adults aged 21 to 51 . Their findings support several questions on the LHS for which we did not have data (e.g., "Did you get any kind of special help in school?", "If you didn't finish school, why did you leave?”, "Have you ever lived on your own?”), as well as day-to-day behavior items. On parallel questions, the Streissguth study found that $65 \%$ of clients received remedial help in reading and arithmetic; $60 \%$ were suspended, expelled, or dropped out of school; more than half (55-60\%) were repeatedly disruptive and had repeated problems getting along with peers; and over $80 \%$ of adults resided in dependent living situations. Spohr and colleagues (2007) similarly found that the effects of PAE 
tended to persist at least into early adulthood, with most of the diagnosed subjects manifesting school, employment, and behavioral problems, as well as an inability to live on their own.

\section{Establishing LHS Psychometrics: Next Steps}

The impetus to develop the LHS was our observation that a subset of women in PCAP struggled disproportionately with the learning and behavioral expectations of inpatient and outpatient substance abuse treatment; we surmised that their difficulties might be associated with alcohol-related brain damage. As we developed the LHS, we were encouraged by the fact that colleagues at substance abuse treatment facilities across the United States were eager to use the screen, even though it did not have established psychometric properties. Therefore, we conducted the present study as a first step toward that goal, presenting information about the screening tool based solely upon data already available. Unfortunately, these data do not include responses to all of the items on the originally-formulated LHS, which limits what can be done at this point in terms of scale development. However, given the positive results of this preliminary study, it is appropriate to consider the next steps necessary to establish LHS psychometrics.

Following the methodology described by Streissguth and colleagues (1998) in their development of the FABS, the next step for LHS development will be a Derivation Study to collect data on the full set of items with a sample large enough to allow assessment of the scale's internal consistency and reliability. If the scale is insufficiently reliable, it will be necessary to add items in order to bolster it, or drop items that are detracting from its reliability. Construct validity will be a primary concern (i.e., does the scale actually focus on consequences of prenatal alcohol exposure?). If the LHS is reliable, results of the measure should correlate with results of other FASD screening measures for concurrent validity (e.g., FABS). When data for the full set of LHS items are available, it will be possible to replicate the ROC analysis for the screening instrument as a whole.

The present sample was in many ways ideal for providing initial confidence that the approach we are taking can differentiate those with a strong likelihood of FASD from those without serious prenatal alcohol exposure. However, because of the relatively high base rates of FASD and PAE in the PCAP sample, it is very likely that this brief screening scale (and also the LHS in its entirety) will perform differently in clinical populations with lower base rates and associated mental health conditions. Thus, it will be desirable to replicate the ROC analysis with populationbased clinical samples as they are studied. Such samples also will provide an opportunity to assess the effects of using different classification cut-points so as to emphasize sensitivity (where the priority is detection of individuals with PAE) or specificity (in settings where it is necessary to be especially selective in making diagnostic referrals based upon the screening instrument). Further, having available a formally established criterion (namely, results of FASD diagnostic evaluations for at least a sizable subset of the sample) will allow for even more precise evaluations of the
LHS's accuracy in terms of its overall classification accuracy, sensitivity, and specificity, as well as determination of optimal cut-points for programmatic purposes. Subsequently, administering the refined LHS to a general population sample (a Normative Study) will allow for further refinements, especially in terms of cut-points for classification of scores into normal, borderline, and clinical ranges, as well as improved estimates of other scale psychometrics, including age-specific norms. Finally, a Test-Retest Study and possibly a Prediction Study (for instance, predicting continuing troubles reflected on the LHS) could contribute in important ways to the formal establishment of the LHS as a standard self-report FASD screen.

\section{Limitations and Recommendations}

As noted earlier, it is understood that self-reported information, especially by individuals with possible brain damage, may produce inaccurate historical data. This is an important study limitation. However, the purpose of the LHS is not to elicit a reliable history, but rather to screen quickly and efficiently for the possibility of brain-based problems that could interfere with treatment success and refer clients with such problems for diagnostic assessment, if possible.

Another limitation has to do with the sample population. Data came exclusively from female clients entering treatment. Thus, results should not be generalized to groups with different characteristics. For example, it will be important to determine if the measure reliably identifies men as well as women in terms of FASD (diagnosed or suspected). Similarly, the measure should be tested on clients entering other kinds of treatment programs, such as mental health therapy.

It is important to note that the arrest history item on the LHS has qualified support in the literature in terms of its link with FASD. For example, while some investigators have reported an increased rate of conduct problems and criminal behavior in adolescents and adults with FASD (Streissguth et al., 2004), others have found no direct link between FASD and criminal behavior (Fast \& Conry, 2009), or no association absent adverse sociocultural influences (Lynch, Coles, Corley, \& Falek, 2003). Thus, research regarding the FASD-crime connection is both limited and contradictory at this point. We include the arrest item on the LHS because the screening tool is designed to be used with clients who are being treated for a substance abuse problem (certainly an adverse sociocultural experience), and a substantial body of research supports a direct connection between substance abuse and criminal behavior (Tripodi \& Bender, 2011).

Another issue that is highly likely to arise while screening individuals for FASD is comorbidity (Bertrand et al., 2004; Famy, Streissguth, \& Unis, 1998; Huggins, Grant, O’Malley, \& Streissguth, 2008; LaDue, Streissguth, \& Randals, 1992; O'Connor \& Paley, 2009; O’Connor et al., 2002; Roebuck, Mattson, \& Riley, 1999; Spohr et al., 2007; Steinhausen, 1996; Steinhausen \& Spohr, 1998). Therefore, it will be very important-especially in 
treatment programs-to identify the possibility of cooccurring conditions and modify treatment approaches accordingly. It also will be prudent, in terms of treatment planning, to differentiate between prenatal and postnatal etiology with respect to cognitive impairment. For example, chronic alcohol abuse by a patient introduces the possibility of self-inflicted brain damage, particularly in the areas of memory, attention, new learning, visual-spatial processing, and executive skills such as concept formation, cognitive flexibility, set shifting, problem-solving, and ability to follow complex demands (Toneatto, 1997). If individuals who screen positive for possible brain-based disability on the LHS are referred for stepwise and costefficient multidisciplinary evaluation, as we advocate, differential diagnosis should be able to identify comorbid mental health conditions that could complicate or derail treatment.

\section{Conclusion}

While full FASD assessment is ideal for individuals who screen positive on the LHS, limited funding often precludes full diagnostic assessment. Minimally in such cases, testing by a neuropsychologist with expertise in FASD would be helpful in identifying cognitive deficits that are likely to affect treatment progress and selecting treatment approaches that would be most effective for the individual.

\section{Acknowledgments}

We extend special thanks to the Parent-Child Assistance Program (PCAP) case managers, clinical supervisors, and women enrolled in the program for their valuable contributions to this work, and to Cara Ernst, M.A. for data management. This work was supported by the State of Washington Department of Social and Health Services (DSHS) contracts \#0965-69005 and \#1165-29940.

\section{References}

Abel, E. L. (1998). Fetal alcohol abuse syndrome. New York, NY, United States: Plenum Press.

Alati, R., Clavarino, A., Najman, J. M., O’Callaghan, M., Bor, W., Mamun, A. A., \& Williams, G. M. (2009). The developmental origin of adolescent alcohol use: Findings from the Mater University study of pregnancy and its outcomes. Drug and Alcohol Dependence, 98, 136-143. doi:10.1016/j.drugalcdep. 2008.05.011

Astley, S. J., Bailey, D., Talbot, C., \& Clarren, S. K. (2000). Fetal alcohol syndrome (FAS) primary prevention through FAS diagnosis: II. A comprehensive profile of 80 birth mothers of children with FAS. Alcohol and Alcoholism, 35, 509-519.

Barr, H. M., Bookstein, F. L., O’Malley, K. O., Connor, P. D., Huggins, J. E., \& Streissguth, A. P. (2006). Binge drinking during pregnancy as a predictor of psychiatric disorders on the structured clinical interview for DSM-IV in young adult offspring. American Journal of Psychiatry, 163, 1061-1065.
Baer, J. S., Barr, H. M., Bookstein, F. L., Sampson, P. D., \& Streissguth, A. P. (1998). Prenatal alcohol exposure and family history of alcoholism in the etiology of adolescent alcohol problems. Journal of Studies on Alcohol, 59, 533-543.

Baer, J. S., Sampson, P. D., Barr, H. M., Connor, P. D., \& Streissguth, A. P. (2003). A 21-year longitudinal analysis of the effects of prenatal alcohol exposure on young adult drinking. Archives of General Psychiatry, 60, 377-385.

Bertrand, J., Floyd, R. L., Weber, M. K., O’Connor, M., Riley, E. P., Johnson, K. A., \& Cohen, D. E. (2004). National Task Force on FAS/FAE. Fetal Alcohol Syndrome: Guidelines for referral and diagnosis. Atlanta, GA, United States: Centers for Disease Control and Prevention.

Boland, F., Burrill, R., Duwyn, M., \& Karp, J. (1998). Fetal alcohol syndrome: Implications for correctional service. Retrieved from http://www.csc-scc.gc.ca

Bookstein, F. L., \& Kowell, A. P. (2010). Bringing morphometrics into the fetal alcohol report: Statistical language for the forensic neurologist or psychiatrist. Journal of Psychiatry and Law, 38, 449-473.

Brown, N. N., Gudjonsson, G., \& Connor, P. (2011). Suggestibility and fetal alcohol spectrum disorders (FASD): I'll tell you anything you want to hear. Journal of Psychiatry and Law, 39, 39-71.

Brown, N. N., Wartnik, A. P., Connor, P. D., \& Adler, R. S. (2011). A proposed model standard for forensic assessment of FASD. Journal of Psychiatry and Law, 38, 383-418.

Centers for Disease Control and Prevention (CDC). (2004). Fetal alcohol syndrome: Guidelines for referral and diagnosis. National Center on Birth Defects and Developmental Disabilities, National Task Force on Fetal Alcohol Syndrome and Fetal Alcohol Effect. Retrieved from http://www.cdc.gov/ncbddd/fasd/ documents/fas_guidelines_accessible.pdf

Clark, E., Lutke, J., Minnes, P., \& Ouellette-Kuntz, H. (2004). Secondary disabilities among adults with fetal alcohol spectrum disorder in British Columbia. Journal of FAS International, 2, 1-12.

Ernst, C. C., Grant, T. M., Streissguth, A. P., \& Sampson, P. D. (1999). Intervention with high-risk alcohol- and drug-abusing mothers: II. 3-year findings from the Seattle model of paraprofessional advocacy. Journal of Community Psychology, 27, 19-38. doi:10.1016/j.ajog.2008.09.871

Famy, C., Streissguth, A. P., \& Unis, A. S. (1998). Mental illness in adults with fetal alcohol syndrome or fetal alcohol effects. American Journal of Psychiatry, 155, 552-554.

Fast, D. K., \& Conry, J. (2009). Fetal alcohol spectrum disorders and the criminal justice system. Developmental Disabilities Research Reviews, 15, 250-257. doi:10.1002/ddrr.66

Goh, Y. I., Chudley, A. E., Clarren, S. K., Koren, G., Orrbine, E., Rosales, T., \& Rosenbaum, C. (2008). Development of Canadian screening tools for fetal alcohol spectrum disorder. Canadian Journal of Clinical Pharmacology, 15, e344-366. 
Grafman, J., \& Litvan, I. (1999). Importance of deficits in executive functions. Lancet, 354, 1921-1923.

Grant, T. M., Brown, N. N., Dubovsky, D., Sparrow, J., \& Ries, R. (2013). The impact of prenatal alcohol exposure on addiction treatment. Journal of Addiction Medicine, 7(2), 87-95. doi:10.1097/ADM.0b013e 31828b47a8

Grant, T. M., Brown, N. N., Graham, J. C., \& Ernst, C. E. (in press). Substance use treatment among women who have a fetal alcohol spectrum disorder or other neurocognitive impairment. International Journal of Alcohol and Drug Research.

Grant, T. M., Ernst, C. C., Streissguth, A., \& Stark, K. (2005). Preventing alcohol and drug exposed births in Washington State: Intervention findings from three parent-child assistance program sites. American Journal of Drug and Alcohol Abuse, 31, 471-490.

Grant, T. M., Huggins, J., Graham, J. C., Ernst, C., Whitney, N., \& Wilson, D. (2011). Maternal substance abuse and disrupted parenting: Distinguishing mothers who keep their children from those who do not. Children and Youth Services Review, 33, 2176-2185.

Grant, T. M., Jack, D. C., Fitzpatrick, A. L., \& Ernst, C. C. (2011). Carrying the burdens of poverty, parenting, and addiction: Depression symptoms and selfsilencing among ethnically diverse women. Community Mental Health Journal, 47, 90-98. doi:10.1007/s10597-009-9255-y

Hellemans, K. G., Sliwowska, J. H., Verma, P., \& Weinberg, J. (2010). Prenatal alcohol exposure: Fetal programming and later life vulnerability to stress, depression and anxiety disorders. Neuroscience and Biobehavioral Reviews, 34, 791-807. doi:10.1016/j.neubiorev.2009.06.004

Hoyme, H. E., May, P. A., Kalberg, W. O., Kodituwakku, P., Gossage, J. P., Buckley, D. G., . . Robinson, L. K. (2005). A practical clinical approach to diagnosis of fetal alcohol spectrum disorders: Clarification of the 1996 institute of medicine criteria. Pediatrics, 115, 39-47.

Huggins, J. E., Grant, T. M., O’Malley, K., \& Streissguth, A. P. (2008). Suicide attempts among adults with fetal alcohol spectrum disorders: Clinical considerations. Mental Health Aspects of Developmental Disabilities, 11, 33-41.

Jones, K. L., \& Smith, D. W. (1973). Recognition of the fetal alcohol syndrome in early infancy. Lancet, 302, 999-1001.

Jones, K. L., Smith, D. W., Ulleland, C. N., \& Streissguth, P. (1973). Pattern of malformation in offspring of chronic alcoholic mothers. Lancet, 1, 1267-1271.

Kodituwakku, P. W. (2007). Defining the behavioral phenotype in children with fetal alcohol spectrum disorders: A review. Neuroscience and Biobehavioral Reviews, 31, 192-201.

Kodituwakku, P. W., Kalberg, W., \& May, P. A. (2001). The effects of prenatal alcohol exposure on executive functioning. Alcohol Research \& Health, 25, 192-198.

LaDue, R. A., Streissguth, A. P., \& Randels, S. P. (1992). Clinical considerations pertaining to adolescents and adults with fetal alcohol syndrome. In T.B. Sonderregger (Ed.), Perinatal substance abuse:
Research findings and clinical implications (pp. 104131). Baltimore, MD, United States: John Hopkins University Press.

Lipinski, R. J., Hammond, P., O’Leary-Moore, S. K., Ament, J. J., Pecevich, S. J., Jiang, Y., . . Sulik, K. K. (2012). Ethanol-induced face-brain dysmorphology patterns are correlative and exposure-stage dependent. PLoS One, 7, e43067. Retrieved from http://www. plosone.org/article/info\%3Adoi\%2F10.1371\%2Fjourn al.pone.0043067

Löser, H., Bierstedt, T., \& Blum, A. (1999). Alkoholembryopathie im erwachsenenalter. Eine langzeitstudie. [Fetal alcohol syndrome in adulthood. A long-term study]. Deutsche Medizinische Wochenschrift, 124, 412-418.

Lynch, M. E., Coles, C. D., Corley, T., \& Falek, A. (2003). Examining delinquency in adolescents differentially prenatally exposed to alcohol: The role of proximal and distal risk factors. Journal of Studies on Alcohol, 64, 678-686.

Mattson, S. N., Goodman, A. M., Caine, C., Delis, D. C., \& Riley, E. P. (1999). Executive functioning in children with heavy prenatal alcohol exposure. Alcoholism: Clinical and Experimental Research, 23, 1808-1815.

Mattson, S. N., \& Riley, E. P. (2000). Parent ratings of behavior in children with heavy prenatal alcohol exposure and IQ-matched controls. Alcoholism: Clinical and Experimental Research, 24, 226-231.

May, P. A., Gossage, J. P., Kalberg, W. O., Robinson, L. K., Buckley, D., Manning, M., \& Hoyme, H. E. (2009). Prevalence and epidemiologic characteristics of FASD from various research methods with an emphasis on recent in-school studies. Developmental Disabilities Research Reviews, 15, 176-192. doi:10.1002/ddrr.68

McLellan, A. T., Kushner, H., Metzger, D., Peters, R., Smith, I., Grissom, G., ... Argeriou, M. (1992). The fifth edition of the addiction severity index. Journal of Substance Abuse Treatment, 9, 199-213.

National Institute on Drug Abuse (NIDA). (2011). DrugFacts: Treatment Statistics. National Institutes of Health, U.S. Department of Health and Human Services. Retrieved from http://www.drugabuse.gov/ publications/drugfacts/treatment-statistics

O'Connor, M. J., \& Paley, B. (2009). Psychiatric conditions associated with prenatal alcohol exposure. Developmental Disabilities Research Reviews, 15, 225-234. doi:10.1002/ddrr.74

O'Connor, M. J., Shah, B., Whaley, S., Cronin, P., Gunderson, B., \& Graham, J. (2002). Psychiatric illness in a clinical sample of children with prenatal alcohol exposure. American Journal of Drug and Alcohol Abuse, 28, 743-754.

Public Health Agency of Canada (2011). Assessment and diagnosis of FASD among adults: A national and international systematic review. Retrieved from http://www.phac-aspc.gc.ca/hp-ps/dca-dea/progini/fasd-etcaf/publications/ad-ed/index-eng.php

Roebuck, T. M., Mattson, S. N., \& Riley, E. P. (1999). Behavioral and psychosocial profiles of alcoholexposed children. Alcoholism: Clinical and Experimental Research, 23, 1070-1076. 
Sparrow, J., Grant, T. M., Connor, P., \& Whitney, N. (2013). The value of the neuropsychological assessment for adults with fetal alcohol spectrum disorders: A case study. International Journal of Alcohol and Drug Research, 2, 79-86. doi: 10.7895/ijadr.v2ie.172.107

Spohr, H. L., Willms, J., \& Steinhausen, H. C. (2007). Fetal alcohol spectrum disorders in young adulthood. Journal of Pediatrics, 150, 175-179.

Steinhausen, H. C. (1996). Psychopathology and cognitive functioning in children with fetal alcohol syndrome. In H.L. Spohr \& H.C. Steinhausen (Eds.), Alcohol, pregnancy and the developing child (pp. 227-246). New York, NY, United States: Cambridge University Press.

Steinhausen, H. C., \& Spohr, H. L. (1998). Long-term outcome of children with fetal alcohol syndrome: Psychopathology, behavior, and intelligence. Alcoholism: Clinical and Experimental Research, 22, 334-338.

Stratton, K. R., Howe, C. J., \& Battaglia, F. C. (1996). Fetal alcohol syndrome: Diagnosis, epidemiology, prevention, and treatment. Washington, DC, United States: National Academy Press.

Streissguth, A. P., Barr, H. M., Kogan, J. \& Bookstein, F. (1996). Understanding the occurrence of secondary disabilities in clients with fetal alcohol syndrome (FAS) and fetal alcohol effects (FAE). Final report to the Centers for Disease Control and Prevention $(C D C)$. Seattle, WA, United States: University of Washington, Fetal Alcohol \& Drug Unit. Rep. No. 9606 .
Streissguth, A. P., Bookstein, F. L., Barr, H. M., Press, S., \& Sampson, P. D. (1998). A fetal alcohol behavioral scale. Alcoholism: Clinical and Experimental Research, 22(2), 325-333.

Streissguth, A. P., Bookstein, F. L., Barr, H. M., Sampson, P. D., O'Malley, K., \& Young, J. K. (2004). Risk factors for adverse life outcomes in fetal alcohol syndrome and fetal alcohol effects. Journal of Developmental and Behavioral Pediatrics, 25, 228238.

Substance Abuse and Mental Health Services Administration (SAMHSA). (2012). Treatment episode data set (TEDS) 2009. Discharges from substance abuse treatment services. Abuse and Mental Health Services Administration. Retrieved from http://www.samhsa.gov/data/2k12/TEDS2009N/TEDS 09DWeb.pdf

Toneatto, T. (1997). Alcohol-produced cognitive deficits: Treatment implications. In S. Harrison, \& V. Carver (Eds.), Alcohol \& drug problems: A practical guide for counsellors. (pp. 439-449). Toronto, ON: Addiction Research Foundation.

Tripodi, S. J., \& Bender, K. (2011). Substance abuse treatment for juvenile offenders: A review of experimental and quasi-experimental research. Journal of Criminal Justice, 39, 246-252.

Yates, W. R., Cadoret, R. J., Troughton, E. P., Stewart, M., \& Giunta, T. S. (1998). Effect of fetal alcohol exposure on adult symptoms of nicotine, alcohol, and drug dependence. Alcohol: Clinical and Experimental Research, 22, 914-920.

\section{Appendix}

\section{Protocol for Administering the Life History Screen (LHS)}

The LHS is a structured screening instrument that can be embedded within a treatment program's existing overall intake protocol so the clinician can observe the pattern of responses within the context of screening for FASD. It begins with the clinician explaining the purpose of the LHS interview, as in the following example:

"While we are talking today, I am going to be asking you questions about your biological family, your history, and things that have happened to you in your life. These questions may seem really personal and, if so, please stop me so I can explain. They are important in order to help me understand the challenges you've had and help me learn more about how we can best help уои."

If the client exhibits resistance or defensiveness, the clinician should reassure him/her that no assumptions or judgments are being made and that the goal of the questions is to help the client succeed in the treatment program.

If the client's responses indicate the possibility of FASD and associated cognitive impairment, the clinician should discuss this immediately, in order to put the interview into a helpful context. The following is an example of a discussion with a client about the possibility that he/she may have an FASD.

"Let's spend a few minutes reviewing what I have learned from you. You told me that there are a lot of people in your family who have had problems with alcohol or drugs, including your mom. You told me that people in your family told you that she drank a lot when she was pregnant with you. As you've grown up, you've had a hard time. You had a tough time in school because things were hard for you and you got into a lot of fights. You've had a hard time keeping a job. And you've been to jail a lot. Did you ever wonder why things were hard for you?"

After giving the client an opportunity to talk at length, the clinician might state the following: "It sounds like life has been pretty hard for you, and one of the reasons might go back to what happened when your mom was pregnant with you. It's possible that her drinking may have changed the way your brain developed. That may be why things are sometimes harder for you than for other people. What do you think about that?"

Depending on how the client responds, it may be important to emphasize that the difficulties she has are not about "intelligence," but rather how her brain uses information and deals with situations. If the client blames or defends her mother, remind him/her that no one is passing judgment on the mother or thinks the mother wanted to hurt the client on purpose. 
The clinician should explain the expectations of the treatment program, asking the client to describe what he/she perceives will be difficult and what might help ensure success. For example, a client with an FASD may state that keeping track of time and appointments is difficult, or that group treatment is overwhelming because of the fast pace. The clinician should reassure the client that they can work together to devise solutions, always with the goal of helping the client succeed in the program.

LHS screening results do not constitute a diagnosis of FASD. Results may signal the need for further assessment and possible FASD diagnostic evaluation, which are very valuable if available. Clinicians should not make the leap from screening to tailoring interventions without knowing the particular strengths and impairments of the individual. We consider a neuropsychological evaluation to be essential for accurately identifying client strengths and impairments, appropriate expectations, and treatment/intervention approaches. In addition, a neuropsychological evaluation may be sufficient for helping the individual to obtain financial and social service benefits, and it may be required as part of the full FASD diagnostic process. 